\title{
Photon Pairs Generated by PRBS Pumping of Silicon Photonic Micro-resonators (1640968- Y2)
}

\section{SHAYAN MOOKHERJEA}

Department of Electrical and Computer Engineering, University of California, San Diego, MC 0407 La Jolla CA 92093-0407 USA

Email:smookher@ucsd.edu

Summary of a Project Outcomes report of research funded by the U.S. National Science Foundation under Project Number 1640968 (Year 2).

\section{Introduction and Goals}

The overall goals of this project to investigate and develop integrated photonpair generation and other types of quantum optics devices that are needed for scalable implementation of measurement-device-independent quantum key distribution (MDI-QKD) and similar quantum communications protocols[1]. Figure 1 shows a conceptual image of a serial communications link used to test the pair generation properties of our silicon microchips. The UCSD sub-project focused on the development of key building blocks for microchips that are costeffective, leverages modern micro-fabrication platforms, reduces operational complexity and improves scalability.

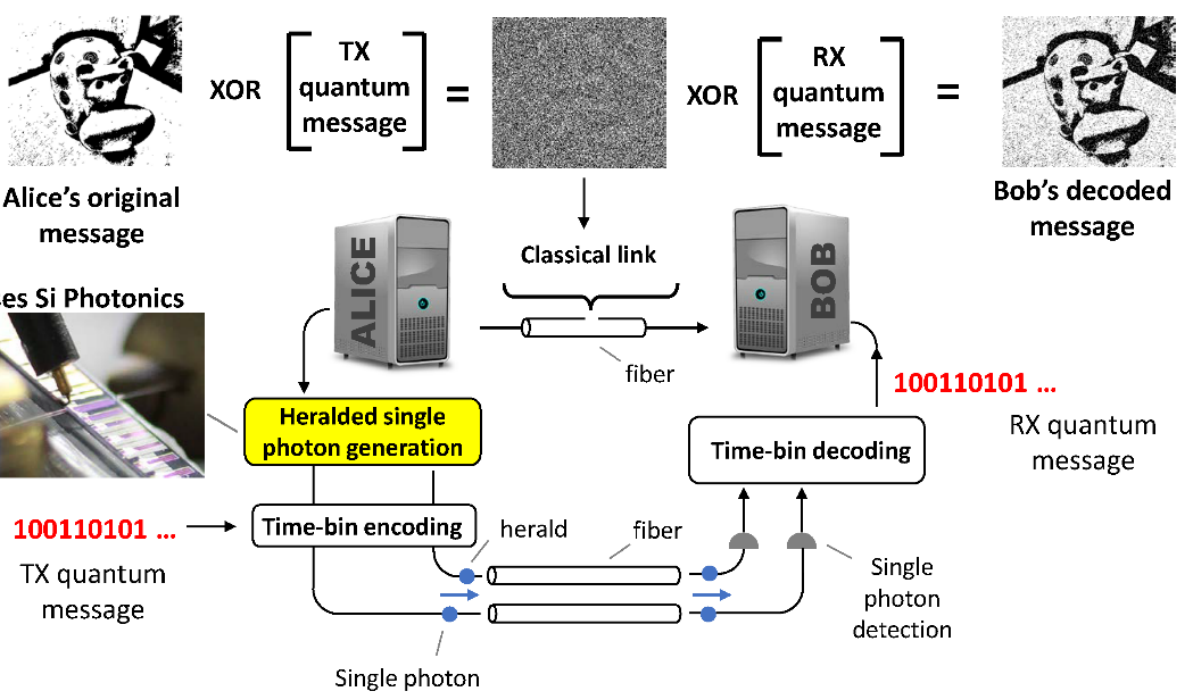

Figure 1 A schematic of a side-channel quantum communications link at $1550 \mathrm{~nm}$ enabled by using a photonic microchip, as a proof-of-concept for photon pair generation and (time-bin) encoding. No separate pump laser is used for the pair generation process - a fraction of the (modulated) light from the classical link is used as the pump.

\section{Activities}


Our research activities this year focused on studying pair sources using integrated silicon photonics technology. Initially, we developed the silicon coupled-resonator optical waveguide structure[2]-[5] which has a strong resonantly enhanced four-wave mixing nonlinearity[6]. Using a Franson interferometer constructed out of conventional optics components, we demonstrated the time-energy entangled nature of the photon pairs generated by our CROW devices around $1550 \mathrm{~nm}$ [7]. However, such devices are sensitive to fabrication disorder[8], and we observed that in practice, an optimized single microring resonator may achieve better performance, and is also a more compact device, which is easier to scale up.

Activities performed this year include:

(i) Demonstration of photon-pair generation using a fraction of a 10 Gbps NRZ data stream (the most common optical signal in the internet) as the optical pump for spontaneous four-wave mixing using a silicon microring resonator[9]. These are based on silicon photonic entangled-pair and heralded single photon generation using a high-Q silicon microring resonator and benefit from improvements we have made in monitoring the microring resonance and thereby stabilizing the pair generation process[10].

(ii) Dual-band entangled photon generation, at both $1310 \mathrm{~nm}$ and $1550 \mathrm{~nm}$ wavelength ranges, using a single silicon microring resonator[11]. This is relevant because our demonstration last year of silicon photonic entangled-pair and heralded single photon generation using hybrid silicon laser [12] was performed at $1310 \mathrm{~nm}$, whereas most of our SFWM experiments are performed at $1550 \mathrm{~nm}$. It is convenient if the same device can be used in either wavelength band.

\section{Impact}

Our silicon photon-pair devices use a milliwatt scale optical pump, which can generated by a laser diode which is inexpensive, compact and energy efficient, especially when compared to traditional Ti:Sapphire lasers that are used to pump entangled photon-pair generation devices in lithium niobate. Moreover, our recent demonstration shows that the pump diode may not be needed in certain cases, where a fraction of a modulated classical optical data stream can be tapped from the network. Only a small fraction of a typical milliwatt-class $10 \mathrm{Gbps}$ NRZ modulated data stream is needed to generate megahertz rates of photon pairs in a high-Q silicon ring. The fact that the pump pulses are modulated using a pseudo random bit sequence (see Fig. 2) does not kill the pair generation process, since the process of spontaneous four-wave mixing is itself random. Not every pump pulse, even in a deterministic stream of pulses such as from a mode locked laser, results in the generation of a pair of entangled photons. In fact, the probability of pair generation is very small (typically 
$0.1 \%$ ), and thus, skipping some pump pulses (on average) has insignificant effect. Ideally, a return-to-zero pulse sequence would be used, so that all the one's have the same width, but in practice, a non-return-to-zero sequence also works, with a reduced efficiency (see Fig. 3). The practical advantages of not having to supply a separate pump laser for each pair generation device are significant and include lower cost, reduced space, and energy savings.

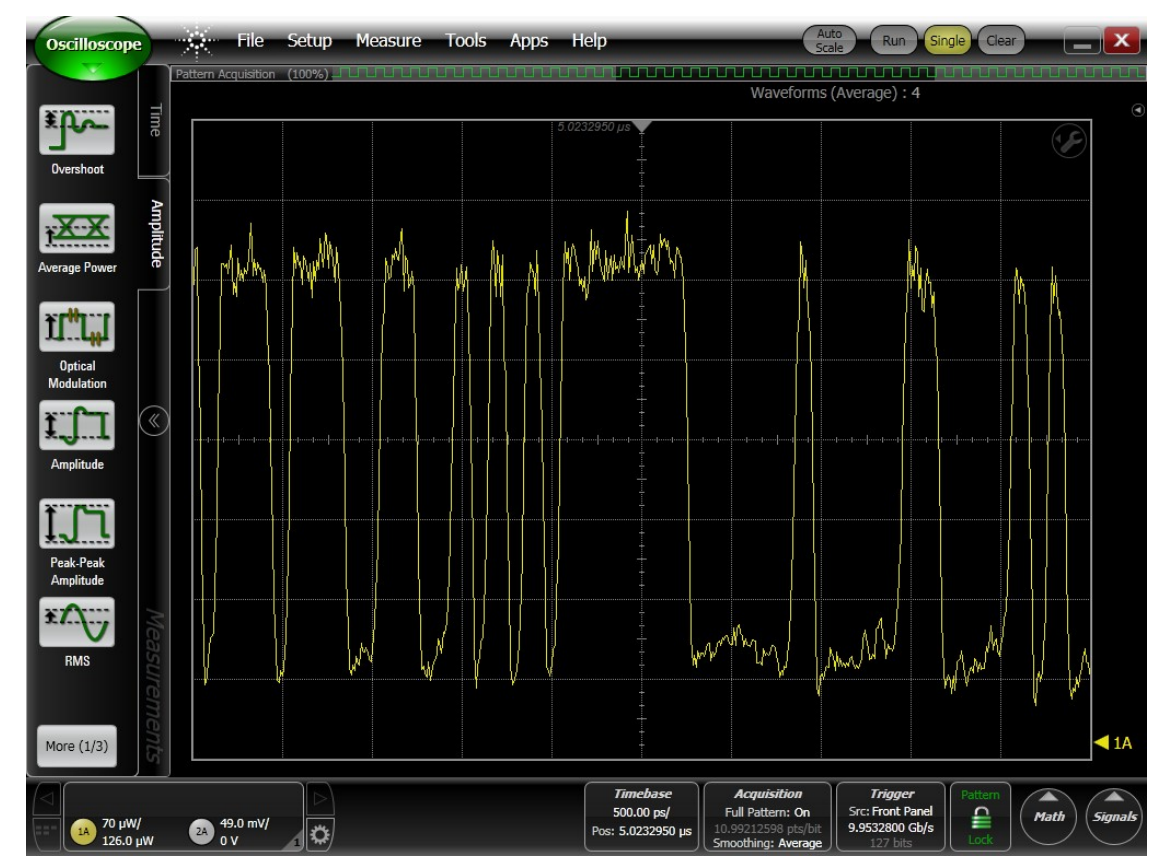

Figure 2 Pseudo-random bit sequence (PRBS) Non-return to Zero (NRZ) modulation at 10 $\mathrm{Gbit} / \mathrm{s}$. The shortest pulses have a duration of about $80 \mathrm{ps}$, which matches the photon lifetime in the microring resonator at the pump wavelength.

Figure 3 shows a comparison between the pair generation process using a $\mathrm{CW}$ pulse and one that uses a pulsed PRBS data sequence. In both cases, a coincidence (C) peak is seen near the left edge, followed by a floor of accidental (A) peaks. This measurement was performed using InGaAs SPAD detectors gated at $50 \mathrm{MHz}$. After normalizing for the different acquisition times $(1000 \mathrm{~s}$ for the trace in the left panel, $170 \mathrm{~s}$ for the trace in the right panel), the coincidence peak for the right panel is seen to be almost exactly one-half (49\%) of that on the left, which is what we expect from a PRBS sequence modulating the pump, which has an equal fraction of 0's (which do not pump the SFWM process and generate pairs) and 1's (which do). 

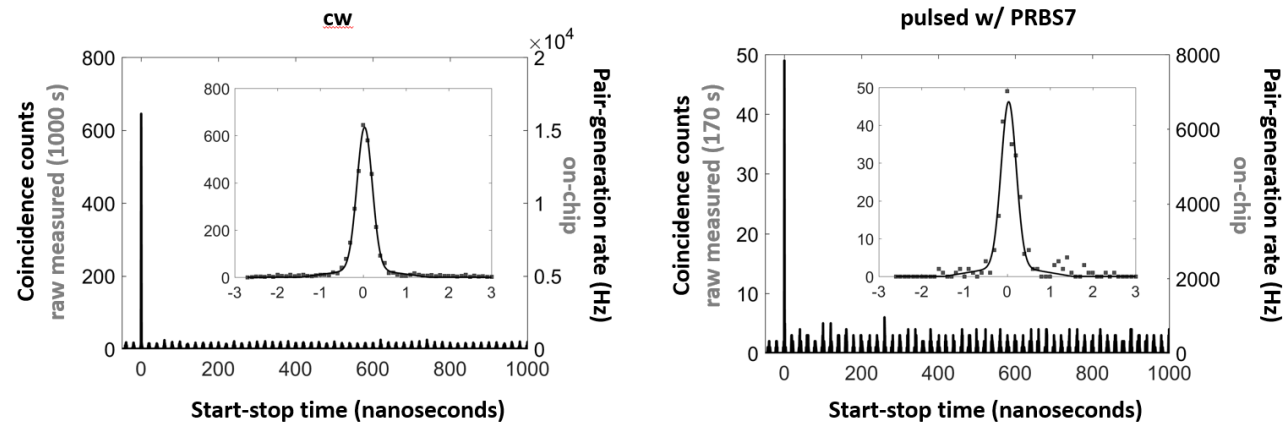

Figure 3 (left panel) Coincidence counts (binned) using a cw pump for an SFWM process in a silicon microring resonator. (right panel) Using a pulse pump with a PRBS NRZ data modulated pulse sequence as a pump.

We have shown that the same microring device can be used at $1310 \mathrm{~nm}$ and $1550 \mathrm{~nm}$ wavelengths. One of the challenges to wideband operation is that the dispersion (i.e., wavelength sensitivity) of the directional coupler between the microresonator and the bus waveguide can be large. However, the same large dispersion issue also affects the coupled-resonator device and can sometimes be used for added functionality[13], [14].

The work of three graduate students at UCSD was supported by this project. They received hands-on training in the fields of electrical engineering, physics, device fabrication and measurement and materials science. Results have been published in peer-reviewed journals and presented at internationally recognized conferences, as well as workshops, seminars, and meetings. Students and researchers from the different groups met and exchanged ideas at major conferences such as CLEO.

\section{Potential Subsequent Extensions}

In traditional approaches to photon-pair generation using spontaneous optical nonlinearities, short pump pulses are usually used (from mode locked lasers) [1]. One of the reasons is to boost the peak intensity, which may not be necessary here since the microring resonators are efficient devices and higher peak powers lead to higher two-photon and free carrier absorption losses. Another reason is to provide accurate timing and synchronization information, like a clock, in a quantum communications link. Although integrated electrooptic modulators can be used to carve pump pulses, they may need a very wide bandwidth that is beyond the capabilities of traditional silicon photonics[15]. The benefit of using modulators is that they can be re-synchronized to an externally obtained timing reference easily, which is necessary between distant parts of a quantum communications network.

One of the challenges of high index contrast integrated photonics using microresonators is the sensitivity to disorder. Effects of disorder in long chains has been studied for the coupled-resonator configuration[16], [17]. However, 
we have also studied ways to study and potentially compensate for disorder after fabrication [18]-[20].

These devices use the efficient all-optical, ultrafast four-wave mixing nonlinearity that occurs in a highly confined waveguide (cross sectional mode area about $0.2 \mu \mathrm{m}^{2}$ ). Physics theory suggests that there are other interesting Kerr nonlinear effects that might occur in appropriately dispersion-engineered devices, such as the generation of bright and dark solitons[21], [22]. These could be alternative ways to shape the pump beam without using electro-optical effects.

\section{Open-Access Reporting Initiative}

PRAISE: This open-access document is provided in support of our PRAISE (Public Report of Activities, Impact and Subsequent Extensions) initiative. What is it? An open-access document shared with the public which describes the research outcomes of publicly-funded projects such as those funded by the U.S. NSF (National Science Foundation).

\section{References}

[1] A. Migdall, S. V. Polyakov, J. Fan, and J. C. Bienfang, Single-Photon Generation and Detection: Physics and Applications, vol. 45. Academic Press, 2013.

[2] S. Mookherjea, "Spectral characteristics of coupled resonators," J. Opt. Soc. Am. B, vol. 23, no. 6, p. 1137, Jun. 2006, doi: 10.1364/JOSAB.23.001137.

[3] S. Mookherjea, D. S. Cohen, and A. Yariv, "Nonlinear dispersion in a coupled-resonator optical waveguide," Opt. Lett., vol. 27, no. 11, p. 933, Jun. 2002, doi: 10.1364/OL.27.000933.

[4] J. R. Ong and S. Mookherjea, "Quantum light generation on a silicon chip using waveguides and resonators," Opt. Express, vol. 21, no. 4, p. 5171, Feb. 2013, doi: 10.1364/OE.21.005171.

[5] M. L. Cooper et al., "235-ring Coupled-Resonator Optical Waveguides," in Conference on Lasers and Electro-Optics 2010, San Jose, California, 2010, p. CTuHH3. doi: 10.1364/CLEO.2010.CTuHH3.

[6] J. R. Ong, R. Kumar, and S. Mookherjea, "Silicon microring-based wavelength converter with integrated pump and signal suppression," Opt. Lett., vol. 39, no. 15, p. 4439, Aug. 2014, doi: 10.1364/OL.39.004439.

[7] R. Kumar, M. Savanier, J. R. Ong, and S. Mookherjea, "Entanglement measurement of a coupled silicon microring photon pair source," Opt. Express, vol. 23, no. 15, p. 19318, Jul. 2015, doi: 10.1364/OE.23.019318. 
[8] S. Mookherjea, J. R. Ong, X. Luo, and L. Guo-Qiang, "Electronic control of optical Anderson localization modes," Nature Nanotech, vol. 9, no. 5, pp. 365-371, May 2014, doi: 10.1038/nnano.2014.53.

[9] C. Ma, X. Wang, and S. Mookherjea, "Photon-pair and heralded single photon generation initiated by a fraction of a $10 \mathrm{Gbps}$ data stream," Opt. Express, vol. 26, no. 18, pp. 22904-22915, Sep. 2018, doi: 10.1364/OE.26.022904.

[10] M. Savanier, R. Kumar, and S. Mookherjea, "Optimizing photon-pair generation electronically using a $p-i-n$ diode incorporated in a silicon microring resonator," Appl. Phys. Lett., vol. 107, no. 13, p. 131101, Sep. 2015, doi: 10.1063/1.4932047.

[11] C. Ma and S. Mookherjea, "Simultaneous dual-band entangled photon pair generation using a silicon photonic microring resonator," Quantum Sci. Technol., vol. 3, no. 3, p. 034001, Jul. 2018, doi: 10.1088/20589565/aab89a.

[12] X. Wang et al., "Photon Pair Generation Using Silicon Photonic Microring and Hybrid Laser," in Conference on Lasers and ElectroOptics, San Jose, California, 2017, p. JTh5C.6. doi: 10.1364/CLEO_AT.2017.JTh5C.6.

[13] S. Mookherjea, "Using gain to tune the dispersion relation of coupledresonator optical waveguides," IEEE Photon. Technol. Lett., vol. 18, no. 5, pp. 715-717, Mar. 2006, doi: 10.1109/LPT.2006.871144.

[14] S. Mookherjea, "Semiconductor coupled-resonator optical waveguide laser," Appl. Phys. Lett., vol. 84, no. 17, pp. 3265-3267, Apr. 2004, doi: 10.1063/1.1719278.

[15] X. Wang, P. O. Weigel, J. Zhao, M. Ruesing, and S. Mookherjea, "Achieving beyond-100-GHz large-signal modulation bandwidth in hybrid silicon photonics Mach Zehnder modulators using thin film lithium niobate," APL Photonics, vol. 4, no. 9, p. 096101, Sep. 2019, doi: 10.1063/1.5115243.

[16] M. L. Cooper and S. Mookherjea, "Modeling of Multiband Transmission in Long Silicon Coupled-Resonator Optical Waveguides," IEEE Photon. Technol. Lett., vol. 23, no. 13, pp. 872-874, Jul. 2011, doi: 10.1109/LPT.2011.2141657.

[17] S. Mookherjea and M. A. Schneider, "Avoiding bandwidth collapse in long chains of coupled optical microresonators," Opt. Lett., vol. 36, no. 23, p. 4557, Dec. 2011, doi: 10.1364/OL.36.004557.

[18] M. L. Cooper, G. Gupta, J. S. Park, M. A. Schneider, I. B. Divliansky, and S. Mookherjea, "Quantitative infrared imaging of silicon-on-insulator microring resonators," Opt. Lett., vol. 35, no. 5, p. 784, Mar. 2010, doi: 10.1364/OL.35.000784. 
[19] S. Mookherjea and H. R. Grant, "High dynamic range microscope infrared imaging of silicon nanophotonic devices," Opt. Lett., vol. 37, no. 22, p. 4705, Nov. 2012, doi: 10.1364/OL.37.004705.

[20] Y. Shen, I. B. Divliansky, D. N. Basov, and S. Mookherjea, "Perfect set-and-forget alignment of silicon photonic resonators and interferometers," in Optical Fiber Communication Conference/National Fiber Optic Engineers Conference 2011, Los Angeles, California, 2011, p. PDPC3. doi: 10.1364/OFC.2011.PDPC3.

[21] B. Crosignani, A. Yariv, and S. Mookherjea, "Nonparaxial spatial solitons and propagation-invariant pattern solutions in optical Kerr media," Opt. Lett., vol. 29, no. 11, p. 1254, Jun. 2004, doi: 10.1364/OL.29.001254.

[22] A. Ciattoni, B. Crosignani, S. Mookherjea, and A. Yariv, "Nonparaxial dark solitons in optical Kerr media," Opt. Lett., vol. 30, no. 5, p. 516, Mar. 2005, doi: 10.1364/OL.30.000516. 